Denis Kapski - Valery Kasyanik - Oleksii Lobashov - Aleksandra Volynets - Oleg Kaptsevich - Andrii Galkin*

\title{
ESTIMATING THE PARAMETERS OF TRAFFIC FLOWS ON THE BASIS OF PROCESSING OF LOCALIZATION DATA ON THE MOVEMENT OF VEHICLES
}

The article describes the method of estimating the parameters of transport flows using the two-fluid mathematical model of Herman-Prigogine and developed and based on the proposed method of estimating the parameters of the system on the basis of passive processing of navigation data on the movement of vehicles. The efficiency of the proposed algorithms, mathematical models for estimating the parameters of road traffic flow and system as a whole was confirmed during its testing using a set of tracks on the main highways of Commonwealth of Independent States.

Keywords: vehicle, traffic flow, navigation system, the parameters of traffic flow, a mathematical model for evaluating traffic flow

\section{Introduction}

At present, traffic congestion in road networks is a topical transport-related issue in most developed countries of the world. First of all, this problem is typical for the street and road networks of cities, where most of the fleet of personal vehicles is concentrated, as well as for key highways and transport corridors, which carry significant volumes of freight and passenger traffic.

Currently, significant experience in implementing measures aimed at solving this problem has been accumulated world-wide. These measures fall into three broad groups:

- measures aimed at increasing the maximum road network capacity (construction and reconstruction of road facilities);

- measures aimed at increasing the efficiency of using the existing road network capacity (improving traffic management);

- measures aimed at regulating the volume and structure of transport demand (introduction of various restrictions on the movement and parking of vehicles; reducing the need of the economy and the population for transport through measures in the field of territorial planning, etc.).

The basis for the development of such measures and the adoption of scientifically grounded solutions for their implementation is the application of mathematical modeling methods for the functioning of the existing and planned transport systems.

Predicting the effect of various measures aimed at managing the road network capacity requires solving various problems of transport modeling:

- predicting the effect of the construction or reconstruction of road facilities requires modeling the distribution of traffic flows over the road network;
- evaluating the effectiveness of measures on improving traffic management as a rule requires modeling the movement of individual vehicles in the traffic flow;

- evaluating the effectiveness of measures on regulating transport demand requires modeling the volume and structure of the need of the population and the economy for driving.

The necessity to use transport models will continue to grow as transport systems evolve (expansion and increase of connectivity of the road network, increased role of multimodal transport, introduction of intelligent transport systems, etc.), and as the already existing problem of congestion in road networks of major transport corridors and cities is worsening.

In order to use certain mathematical models to provide decision-making on the management of transport flows, it is necessary to correctly choose the criteria and methods for assessing traffic flows.

2. Choosing the optimal criteria for assessing traffic flows and the method for mathematical modeling of transport systems

When estimating street and road networks, a variety of tasks and situations may arise. This results in the need to use a number of partial and integral criteria for assessing traffic flows [1].

Partial criteria according to the nature of use can be divided into those used only as descriptors and those used as parameters of the management process. The latter include the average and total delays, the queue length, the leg length minus the queue length.

\footnotetext{
* 'Denis Kapski, ${ }^{2}$ Valery Kasyanik, ${ }^{3}$ Oleksii Lobashov, ${ }^{4}$ Aleksandra Volynets, ${ }^{5}$ Oleg Kaptsevich, ${ }^{6}$ Andrii Galkin

${ }^{1}$ Department of Transport Systems and Technologies, Automotive and Tractor Faculty, Belarusian National Technical University, Minsk, Belarus ${ }^{2}$ Brest Technical University, Belarus

${ }^{3}$ Department of Transport and Logistics, O. M. Beketov Kharkiv National University of Urban Economy, Ukraine

${ }^{4}$ State Enterprise Belarusian Road Scientific Research Institute, Belarus

${ }^{5}$ Department OJSC „AGAT - Control Systems“, company Geographic Information Management System, Belarus

${ }^{6}$ Department of Transport and Logistics, O. M. Beketov Kharkiv National University of Urban Economy, Ukraine

E-mail: galkin.tsı@gmail.com
} 
The most significant criterion for the economic evaluation of the state of the traffic management is often considered to be the transport operation of the street and road network.

Another criterion is the stability of the street and road network functioning. This indicator is defined [2] as a property allowing to not reduce its capacity as a result of full or partial failure of its individual elements. Failure is considered as a change in road traffic conditions, which results in this element of the street and road network being partially or completely excluded from the transport process.

Criteria based on the magnitude of the delay and the queue length are also singled out. The duration of the average delay of the vehicle has been widely used as a criterion for optimizing traffic control at a separate intersection. It has been determined that the average delay closely correlates with such indicators as the total delay, queue length, traffic intensity, parameters of the traffic control mode.

The queue length means the number of vehicles in the queue or its length in linear units. This parameter is closely correlated with the average and maximum delays, traffic intensity, parameters of the traffic control mode, and affects such indicators as traffic speed, number of starting and braking per unit of length.

The queue length can be used as an indicator of the degree of saturation, comparing with the queue length passed per cycle. When the state of saturated flows is reached, the queue length and the associated indicators are considered to be the most acceptable for network management. In this case, the task of management is to minimize the probability of congestion.

The cost effectiveness of road traffic is estimated according to several criteria, the most important are the specific delay and the specific stop calculated per vehicle [2-4].

The density of street and road networks is defined as the ratio of the total length of streets and roads to the area size. The density of street and road networks and their traffic load indicators were considered in the works of A.V. Sigayev, S.A. Vaksman and a number of other authors. Typically, the subject of the research is statistical data that include the following indicators: the density of street and road networks, the length of streets and roads per capita, the number of registered vehicles per $1 \mathrm{~km}$ of streets and roads, the annual mileage of vehicles per $1 \mathrm{~km}$ of streets and roads, etc.

A number of researches are devoted to establish a connection between the density and capacity indicators [5]. The most important drawback of the density indicator and its modifications is the absence of any specific information about any street and road sections. In general, the above-mentioned density indicators are descriptors and give only a general assessment of the state of the network.

When using partial criteria, the question inevitably arises as to which indicators can be given preference and in what cases. It is impossible to find an unambiguous answer to this question.

However, it can be said with sufficient certainty that a significant number of specialists prefer the average delay value of vehicles as the most objective indicator of the quality of traffic control and management.

In case of heavy traffic flows, it is most expedient to estimate traffic conditions by the magnitude of the vehicle queue at the intersection or according to the indicators based on it:
- the queue length to the leg length ratio;

- the leg length minus the queue length.

A constant search for universal integral criteria is made that would allow evaluating both the quality of road traffic in general and the quality of its individual properties, since it is impossible to do this with the help of partial criteria.

D. Drew [6] proposed a valuating integral quality criterion for road traffic - Level of Service (LOS). The LOS is associated with such factors as traffic safety, operating speed, driving comfort and convenience, freedom of maneuvering, flow interruption, travel costs, etc. The usage of this criterion covers all stages of working with street and road networks - planning, design, and operation. Currently, the LOS criterion is used to estimate traffic conditions in both street and road network modeling programs and in highly specialized intersection and junction design programs. One of the drawbacks of the considered criterion is its difficulty and qualitative assessment as a whole, and sometimes the impossibility of quantitative assessment of the effectiveness of individual solutions.

Yu. A. Vrubel [7] proposed a new valuating integral criterion - "losses in road traffic". The losses are understood as the socioeconomic value of the unenforced costs of the movement process.

This criterion is applicable for assessing the quality of both road traffic in general and its individual properties. Quality assessment is carried out in monetary terms, which makes it possible to compare not only the quality of individual properties of road traffic, but also the costs of its accomplishment. This fact makes the comparison very clear and allows easily and quickly optimizing solutions for traffic management according to the criterion of loss minimization.

The variation of the traffic flow operating speed can be viewed as an integral criterion [8]. A significant variation of speeds is a distinctive feature of modern traffic on street and road networks of cities. The speed can range from $60 \mathrm{~km} / \mathrm{h}$ allowed within the city limits to $5-10 \mathrm{~km} / \mathrm{h}$ or less established in cases of congestion. The variation of the traffic flow operating speed evaluates the influence of a whole set of factors in a wide range of their changes, starting from free-flow conditions and ending with congestion situations. Thus, it is the integral criteria that are the most applicable to solving problems arising from the evaluation of the street and road network. When using integral criteria for assessing the quality of traffic management, it is inevitable that methods of mathematical modeling should be applied based on macroscopic models of traffic flow. In this regard, the integral criterion for assessing the quality of traffic management is developed on the basis of macroscopic models of traffic flow.

Historically, the first macroscopic model of the single-lane traffic flow was a model later named the Lighthill-WhithamRichards model [9-10], in which the vehicle flow is regarded as a flow of a one-dimensional compressible fluid.

The Lighthill-Whitham-Richards model assumes that:

- there is a one-to-one relationship between the speed and the linear density of the flow;

- the law of conservation of mass - number of vehicles is fulfilled.

Another version of the Lighthill-Whitham-Richards model was proposed by Tanaka in 1963 [11]. 
The Tanaka model assumes that the vehicle speed cannot exceed a certain maximum value, and in view of this the density of the single-lane traffic flow is calculated. This model plays an important role in the study of traffic flows in terms of traffic safety.

The model developed by Whitham in 1974 takes into account the fact that drivers reduce speed when the density of traffic flow moving in front of them increases, and increase speed when the density decreases.

The Payne model [12] does not raise any assumptions about the dependence of speed on density, and is written as a conservation law.

Several drawbacks of the Payne model and many of the models subsequently proposed were indicated by C. Daganzo [13-14]. It was shown that with strong spatial in homogeneities of the initial conditions, negative speed values can appear (the congestion "dissipates back" as a result of viscosity influence). For certain parameter values, densities exceeding the maximum permissible values can occur ("bumper to bumper"). Moreover, according to these models, the vehicle movement is significantly influenced by vehicles located behind, which in the case of a single lane is hardly possible in the real-life traffic flow.

One of the macroscopic model varieties is the two-fluid mathematical model of Herman-Prigogine. This traffic flow model considers the nonlinear dependencies between the specific travel time and the specific time of delays expended per unit of distance. The scope of use of this model is the street and road network or its sections.

Due to the fact that the kinetic theory studies multilane traffic, Herman and Prigogine put forward the theory of two flows of city traffic. Vehicles in the traffic flow are divided into two groups - moving vehicles and stopped vehicles. The latter include vehicles stopped in the flow itself, i.e. vehicles stopped at intersections, stopped due to a regular congestion, stopped due to interference to traffic, etc., but exclude out-of-traffic vehicles, for example, parked vehicles.

An important property of the macroscopic model of Herman and Prigogine is that two different traffic operating modes can be displayed [15]. These are individual and collective flows, which are functionally dependent on the concentration of vehicles - flow density. With a low flow density, traffic moves in the individual flow mode. When the flow density increases, traffic starts moving in the collective flow mode. In this case, the flow becomes largely independent of the desires of individual drivers in the choice of the driving mode.

The two-flow model is based on two initial assumptions:

- the average operating speed along the street and road network is proportional to the fraction of vehicles in motion;

- the duration of delays of a vehicle moving along the street and road network is proportional to the number of vehicles stopped at a given time.

The studies confirmed the provisions of the two-fluid mathematical model of Herman-Prigogine [16]. Simultaneously, it was established that urban street networks can be characterized by two parameters of the model $-n$ and Tm. These parameters were calculated on the basis of experimental data obtained from surveys of cities around the world.

The Herman-Prigogine model is very attractive for practical use, as it is easily applicable when conducting regular surveys of traffic conditions in comparison with other macroscopic models. The uniqueness of the model is that when assessing the impact of the street and road network load on traffic conditions, it is not necessary to determine the load level, i.e. to determine the traffic flow intensity and the capacity of the elements of the street and road network. To assess the $\mathrm{n}$ and Tm parameters, only the data on specific indicators of the travel time $\mathrm{T}$ and the standing time Ts are needed.

The Herman-Prigogine model can be applied:

- to compare street and road networks of different cities or to compare sections within one street and road network;

- to compare the peculiarities of behavior of drivers and the movement of certain types of vehicles;

- to give a detailed assessment of the influence of geometric and other parameters of the street and road network on traffic conditions;

- in the modeling of traffic flows in order to assess the projected traffic conditions.

\section{Description of the traffic flow model}

The parameters used in the Herman-Prigogine model [15] consisting of two flows (moving and standing vehicles) represent the average data determined on the scale of the whole street and road network for a given period of time.

In accordance with the above provisions, the traffic flow in the street and road network at any time consists of two parts: $f r$ moving vehicles; $f_{S}$ - standing vehicles. In this case, the condition $f r+f_{S}=1$ is observed, and the fraction of standing vehicles $f_{S}$ is determined by the ratio:

$f_{s}=\frac{1}{T_{S}}$,

where $T_{s}$ - specific time expenditure caused by delays, $\min / \mathrm{km}$.

The average specific time expenditure $T(\mathrm{~min} / \mathrm{km})$ is the sum of the average specific travel time $T_{r}(\mathrm{~min} / \mathrm{km})$ and the specific delay time $T_{s}(\mathrm{~min} / \mathrm{km})$ :

$T=T_{r}+T_{s}$.

The average speed of moving vehicles $V r$ is defined as the product:

$V_{r}=V_{m} \cdot f_{r}^{n}$,

where $\mathrm{V}_{\mathrm{m}}$ - the average maximum operating speed in the street and road network or in its considered section;

$n$ - an indicator characterizing the quality of functioning of the street and road network or its considered section, which indicates how the speed decreases in the street and road network as the load increases.

Taking into account the delays, the average operating speed in the street and road network or its considered section $V$ is calculated as

$V=V_{m} \cdot f_{r}^{n+1}$. 
Taking into account the balance equation $f_{r}+f_{s}=1$, Equation (4) can be represented in another form:

$$
V=V_{m} \cdot f_{r}^{n+1}=V_{m} \cdot\left(1-f_{s}\right)^{n+1},
$$

Taking the travel time per unit of length to be $T$, the travel time per unit of length to be $T_{r}$, and the average delay time for the passage of a section of the unit length $T_{s}$, we obtain the relations:

$T=\frac{1}{V}$;

$T_{m}=\frac{1}{V_{m}}$,

where $V$ - the average operating speed in the street and road network including the delays;

$T_{m}$ - the average minimal time for the passage of a section of the unit length.

The $T_{m}$ parameter characterizes the minimum specific time expenditure for moving in the free conditions, i.e. at a very low level of network load, in which there is no interaction between vehicles in the flow.

In turn, the $\mathrm{n}$ parameter, called the Herman-Prigogine criterion, reflects the influence of the load level on the decrease in the operating speed of traffic flows. It can be considered as an indicator of the quality of service of traffic flows in the street and road network.

The second initial provision of the two-fluid model asserts that the duration of delays of a vehicle moving along the street and road network is proportional to the number of vehicles stopped at a given time. In accordance with this, the second assumption of the model is mathematically represented as

$$
f_{s}=\frac{T_{s}}{t}
$$

Equation (5) can be reformulated in order to estimate the travel conditions according to the specific time expenditure as follows:

$$
\begin{aligned}
& T=T_{m}\left(1-f_{s}\right)^{-(n+1)} ; \\
& T=T_{m}\left(1-\frac{T_{s}}{T}\right)^{-(n+1)} ; \\
& T_{r}=T_{m}^{\frac{1}{(n+1)}} \cdot T^{\frac{1}{(n+1)}} .
\end{aligned}
$$

The general formula of the Herman-Prigogine model becomes as follows:

$$
T_{s}=T-T_{m}^{\frac{1}{(n+1)}} \cdot T^{\frac{1}{(n+1)}},
$$

The model and the Herman-Prigogine criterion $n$ represented above make it possible to obtain a systematic assessment of the transport situation in the street and road network as a whole, i.e. to quantify the sensitivity of traffic conditions to a change in the load of the street and road network.

The logarithmic transformation of Equation (12) is as follows:

$$
\ln T s=\frac{1}{(n+1)} \cdot \ln T_{m}+\frac{n}{(n+1)} \cdot \ln T,
$$

In order to use linear regression, Equation (13) is transformed as follows:

$\ln T s=\ln T_{m}+n\left(\ln T-\ln T_{m}\right)$.

The linear dependence equation simplifies the procedure of regression analysis and allows using standard statistical methods for estimating the traffic flow parameters.

The classification of road networks based on the parameters included in the Herman-Prigogine model allows predicting the traffic speed and the time expenditure for travel at any given section of the road network. The classification can be constructed, for example, using the clustering analysis of the experimentally established values of the travel time parameters $\mathrm{T}$ and the standing time $T_{s}$.

\section{Implementation of the traffic flow parameter assessment system}

An integrated assessment of the quality of traffic management can be made on the basis of the Herman-Prigogine model using the data obtained from navigation equipment.

Satellite monitoring of transport is used for dispatch control and management of passenger transport, for solving problems of transport logistics in transportation management systems and automated fleet management systems [17]. Satellite monitoring of transport is a system for monitoring mobile objects, which is built on the basis of satellite navigation systems, cellular and/ or radio communication equipment and technologies, computer technology, and digital maps [18].

The principle of monitoring is to track and analyze the spatial and temporal coordinates of a vehicle. Two monitoring options are available: "on-line" - with remote transfer of coordinate information, and "off-line" - information is read upon arrival at the dispatch center. A mobile module is installed on the vehicle, which consists of the following parts: a satellite receiver, storage and transmission modules for coordinate data.

Data on specific indicators of the travel time and standing time can be collected in an active way, using a specially equipped vehicle for driving through the examined sections of the street and road network.

A passive system can also be applied, in which specific indicators are calculated on the basis of navigational data collected from various vehicles participating in road traffic. Such data can be obtained from various sources, for example, from transportation company servers. Using a passive approach to data collection allows increasing the accuracy of the system, since more data can be examined that are collected from different vehicles controlled by different drivers.

The passive experiment methodology, along with the use of the Herman-Prigogine model, allows assessing the traffic flow parameters and constructing a decision-making support system for controlling transportation, selecting traffic routes, and assessing possible changes in the transport system.

Using the two-fluid model of Herman-Prigogine, a software system has been implemented that allows making quantitative assessment of traffic parameters using navigation data, as well as 


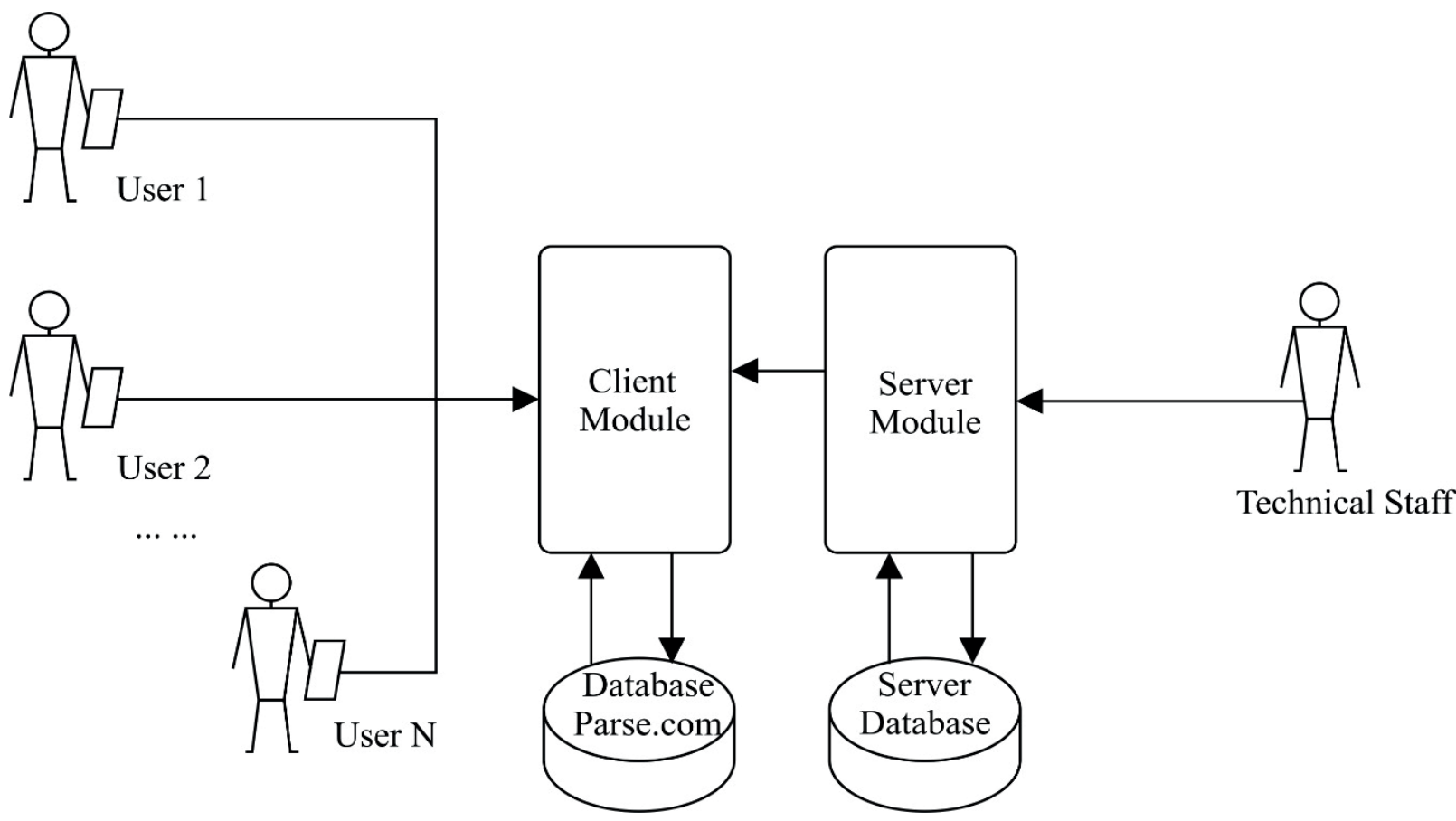

Figure 1 System architecture
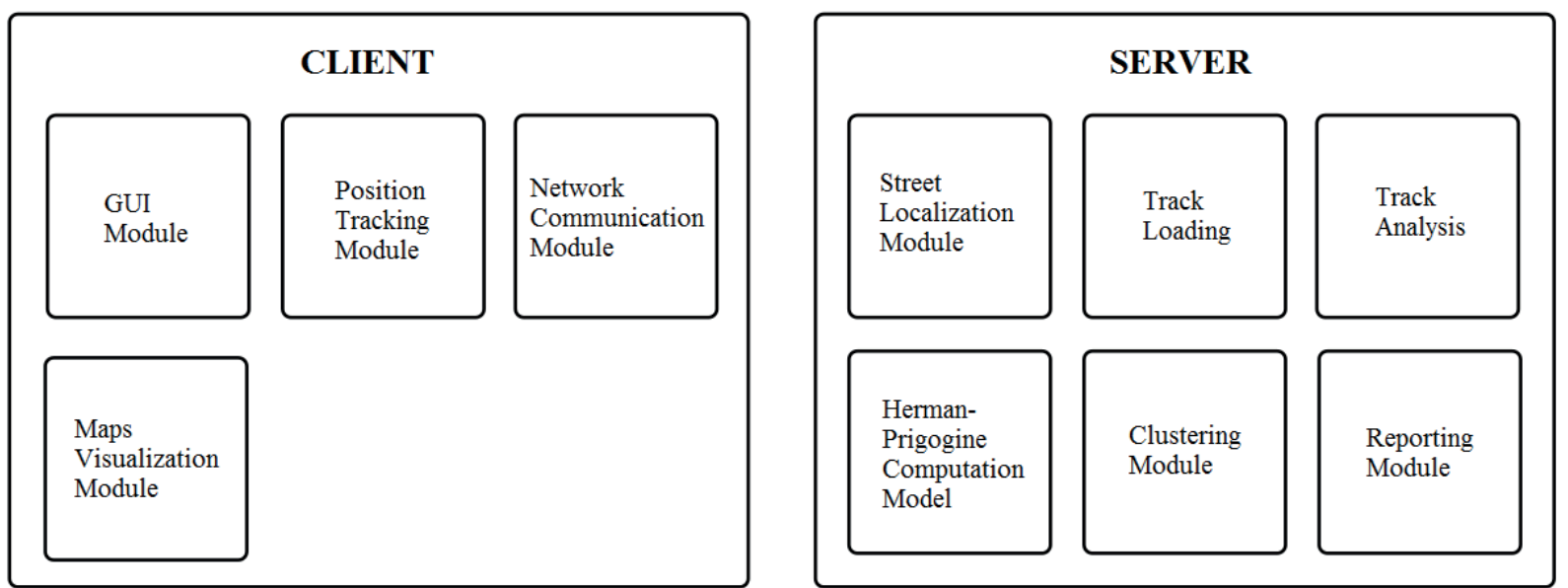

Figure 2 Composition of the client and server subsystems

their visualization using the Google Maps geographic information system.

The software system is designed to collect and analyze navigational data with further application in the assessment of traffic flow parameters on the basis of the two-fluid model of Herman-Prigogine [16, 19, 20]. The data are collected using a large number of navigation modules installed on vehicles or smartphones of users. Data analysis is performed centrally on a high-performance computer, as it requires a considerable amount of calculations.

The software package proposed for the solution of these tasks is implemented on the client-server architecture basis (Figures 1 and 2). Thus, data received from clients are transferred to the server end for further storage, as well as for processing and analysis of these data.

The client end is represented by two types of devices a built-in navigation module integrated in the vehicle or a mobile application based on the Android OS. The built-in equipment automatically sends data on the vehicle location to the transportation company, which performs preliminary data collection with subsequent provision to the system under consideration. The second case is a client application installed on the user's mobile device.

In order to send tracks, users must have the GPS receiver activated on the smartphone or the vehicle, and data transfer must also be enabled. When driving over a certain period of time, users receive their current location. Also, an attempt is made to obtain the name of the street for the current coordinate. The client end of the application analyzes this information, calculating the following parameters on its basis: time, speed, coordinates.

User data are sent to the cloud server Parse.com for further storage and exported in the required format. The Parse.com library was used to work with data for Parse.com. 
The implementation of the client end is represented by a mobile software system for data collection. This system was developed for the Android platform using the Java programming language. The user interface of the system was developed in accordance with the requirements of Google [4] for the interface of Android applications.

The server end of the system is a web application. The web application is used for:

- loading tracks from a file;

- processing tracks according to the Herman-Prigogine model;

- visualizing the obtained results with overlay on the Google Maps cartographic service [21];

- clustering sections of the street and road network according to the parameters of the Herman-Prigogine model.

After preliminary processing and storage in the cloud service, the data are uploaded as a file for further analysis. The file in the CSV format is sent to the server end of the system. The server end of the system at this stage receives all tracks from the file, processes and verifies them. The file in the CSV format containing tracks is sent to the input for analysis. After receiving all tracks for a particular street, the number of tracks, the standing time, and the average speed for this section of the road are determined. Every parameter is assigned a threshold value in advance, on the basis of which it is determined whether a track belongs to the group of unreliable tracks.

After passing the verification, the data are sent to the Herman-Prigogine module for calculating parameters. The following algorithm is used to implement the Herman-Prigogine model. All tracks for a particular street are selected from the track database, and information on time and speed at each point in time associated with obtaining of the location is extracted from them. Information on time allows calculating the total travel time, and the speed allows determining how much of this time is spent standing. Thus, two components are obtained, which are necessary for the Herman-Prigogine model. After receiving the data, a linear regression is constructed using the total time and the time in motion in order to solve an equation of the $\mathrm{y}=\mathrm{kx}+\mathrm{b}$ type. After solving the linear regression, the $\mathrm{k}$ and $\mathrm{b}$ parameters are calculated, which are used for calculating the parameters of the Herman-Prigogine model.

After calculating the parameters of the Herman-Prigogine model, the data are transferred to the clustering module input. The FOREL algorithm [22] is used for clustering the obtained results. This algorithm is based on the idea of combining objects into one cluster in the areas with the most concentration. This algorithm was chosen because it does not need specifying the number of clusters in advance. After clustering of the entire sample, the application displays the charts and routes on the map, and then compiles a report in the TXT format.

The implementation of the server end is represented by a special system developed for the web platform [23-25]. Python was used for the development as the main programming language. The Flask framework [26] was used for displaying in the web environment. The cartographic service Google Maps v3 [27] was used in this system. The JavaScript programming language [23] was used for manipulating the map: constructing points and routes. ORM SqlAlchemy [28] and the SQLite database management system [25] were used for working with the database.
The use of this DBMS is rational within this work in view of the mobility of deployment and maintenance. In the future, when developing this system, it will be necessary to use more powerful DBMS, as the increased amount of data will lead to limitations of the existing solution.

Thus, the hardware of the software package for assessing traffic flow parameters includes:

- mobile device for collecting traffic information - a navigation module integrated in the vehicle or a mobile device with a GPS receiver (Android OS 4.0 and higher) with the data transfer function enabled;

- software products of transportation companies that provide navigation data in the CSV format;

- automated workstation (AWS) of the operator that includes a personal computer running the Linux family operating system. The virtual environment was used to develop the AWS, as well as the Flask framework [29], and the web application was developed using the Python programming language [30].

- SDK Parse.com [31] for Android OS - a library that simplifies the work with the Parse.com platform on mobile devices;

- Parse.com cloud service [31] for storing data transmitted from customers.

\section{Testing the software package for the assessment of traffic flow parameters using experimental data}

The working efficiency of the proposed algorithms, mathematical models for the assessment of traffic flow parameters, and the system as a whole was confirmed during its testing, using a set of tracks on the main highways of Belarus. Similar calculations were made for the city of Minsk, where streets that function at the limit of their capabilities were identified.

To verify the functioning of the Herman-Prigogine model as part of the software package for the assessment of traffic flow parameters, the routes received from one of the transportation companies of the Republic of Belarus with the volume of $5,000,000$ entries were used. A list of tracks for each street or route was used, which was obtained beforehand. Next, it was necessary to calculate the parameters of the Herman-Prigogine model for each street.

The parameters were calculated as follows:

1) two types of time were distinguished for each track: total travel time and standing time. In order to determine what state the vehicle was in - moving or standing, a speed limit of $5 \mathrm{~km} / \mathrm{h}$ was adopted. If the speed was below this limit, the vehicle was considered standing, and if the speed was higher, the vehicle was considered moving;

2) after receiving the time of all tracks, the data was sent to the Herman-Prigogine model, after which the result was obtained. The result is a report file that contains the following information for each route:

- route number - position number of the route in the list of streets or routes;

- address - street, city, country for the current route;

- starting point and end point - route boundary; 


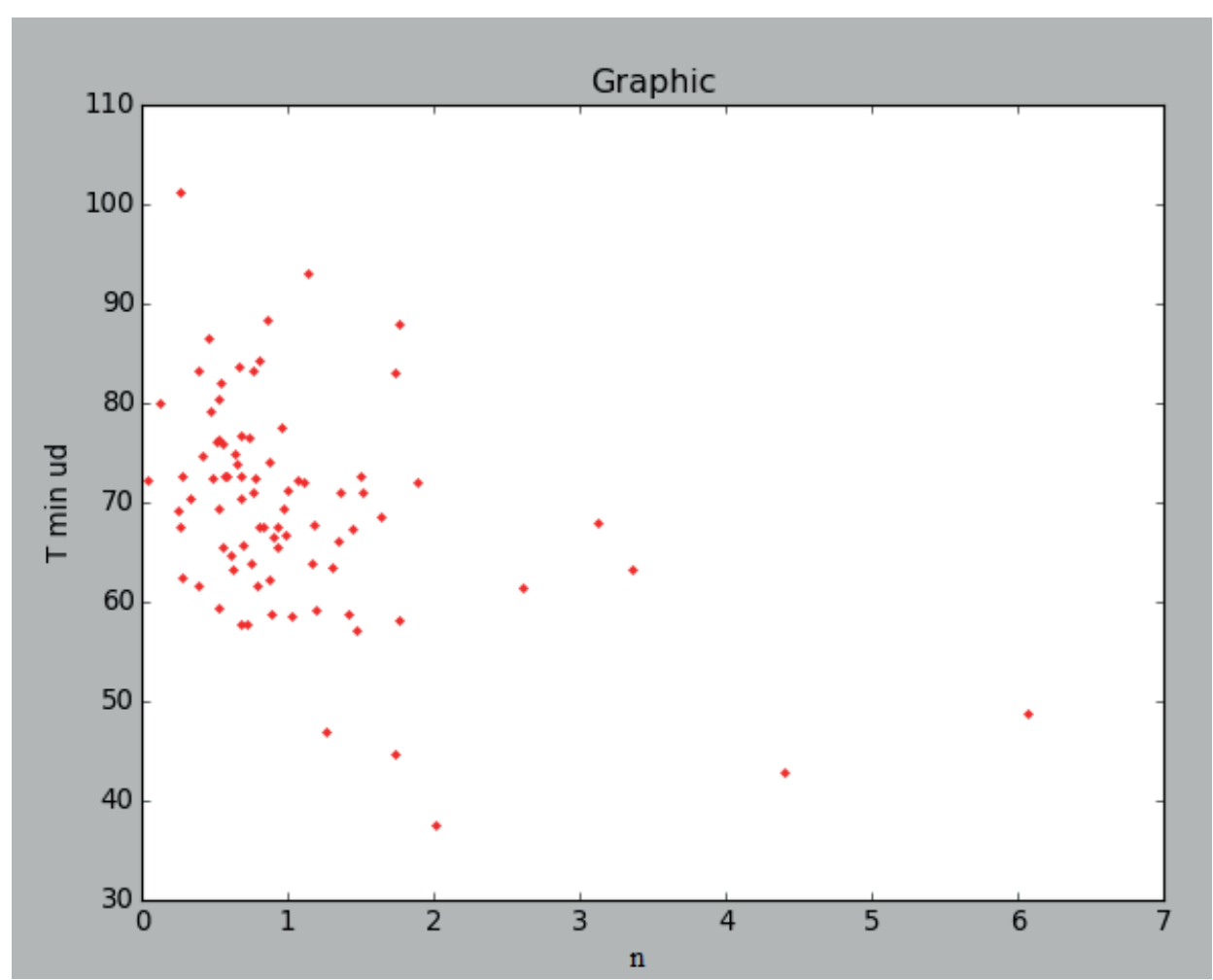

Figure 3 Visualization graph of calculation results according to the Herman-Prigogine model

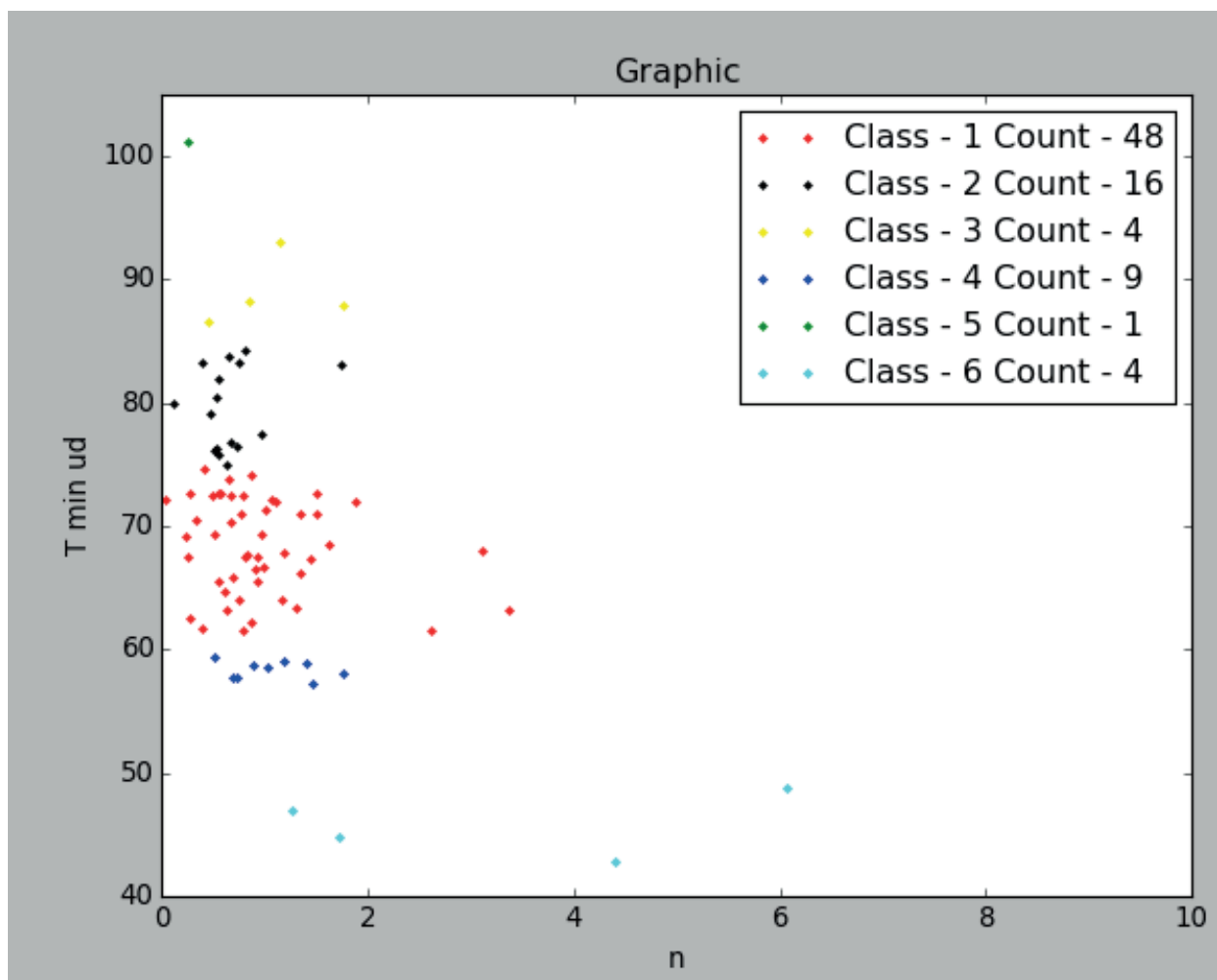

Figure 4 Clustering of routes with a radius of 7 units

- straight-line distance - distance between points in a straight line;

- road distance - distance between points along the road in compliance with traffic rules on this route; speed - minimum average speed obtained as a result of the analysis;

- table with intermediate data - table containing all the tracks for this route in the form of the total travel time, 
Table 1 Characteristics of the obtained clusters

\begin{tabular}{ccccc}
\hline $\begin{array}{c}\text { Cluster } \\
\text { number }\end{array}$ & $\begin{array}{c}\text { Number of } \\
\text { streets }\end{array}$ & Range $n$ & Range $T$ & Brief description \\
\hline 1 & 48 & {$[0.04 ; 3,36]$} & {$[61.51 ; 74.70]$} & Moderate response to increased street load \\
2 & 16 & {$[0.12 ; 1.74]$} & {$[74.91 ; 84.30]$} & Weak response to increased load \\
3 & 4 & {$[0.45 ; 1.76]$} & {$[86.60 ; 93.09]$} & Weak response to increased load \\
4 & 9 & {$[0.52 ; 1.76]$} & {$[57.21 ; 59.39]$} & Weak response to increased load \\
5 & 4 & {$[1.26 ; 6.07]$} & {$[42.86 ; 48.76]$} & Maximum response to increased load \\
6 & 1 & {$[0.26 ; 0.26]$} & {$[101.17 ; 101.17]$} & No response to increased load \\
\hline
\end{tabular}

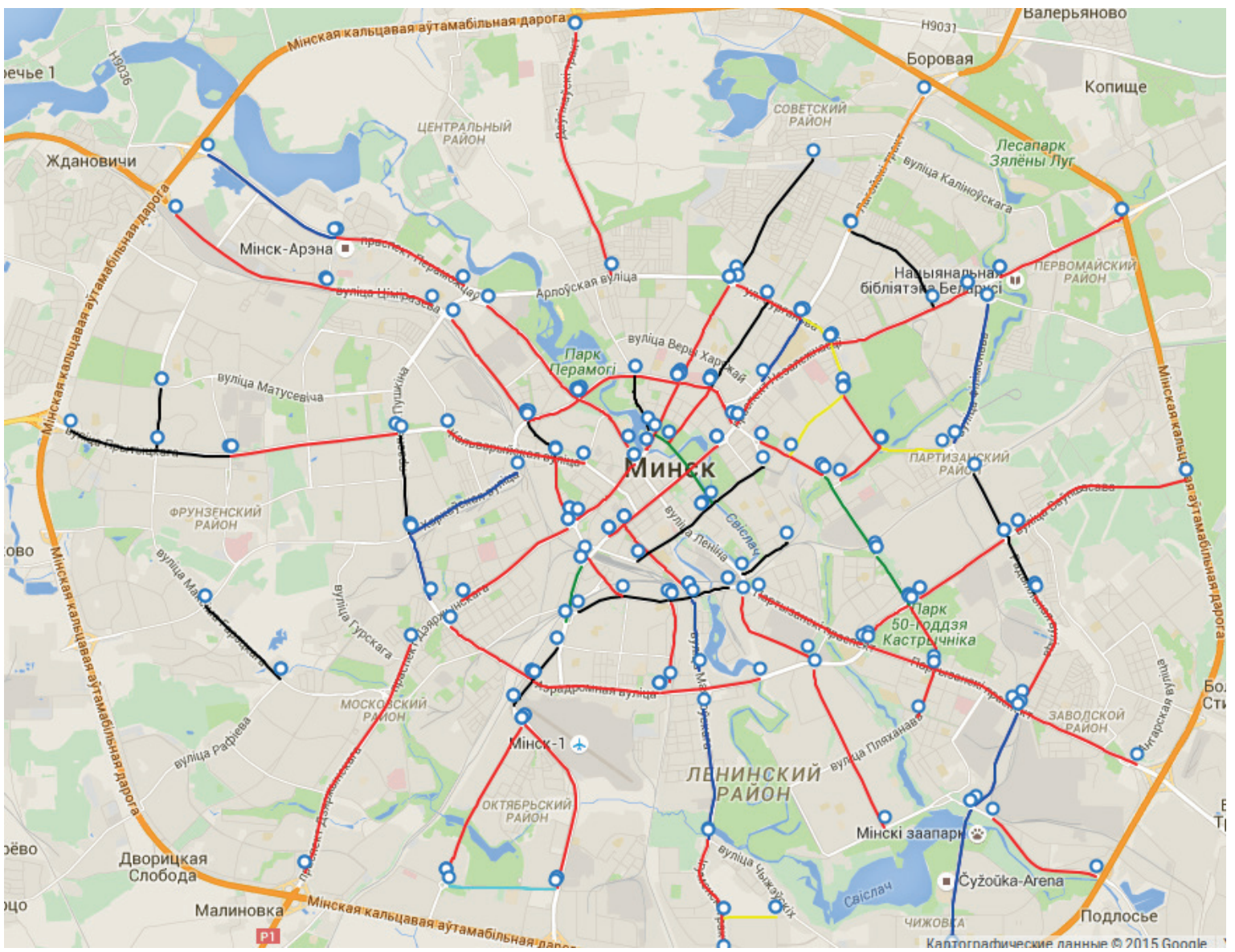

Figure 5 Color differentiation of routes by classes

as well as the standing time. The table also contains intermediate computational values;

- $\quad$ adjustable parameters for $y=k x+b$-adjustable parameters $k$ and $b$ obtained during solving of the linear regression;

- parameters of the Herman-Prigogine model: the HermanPrigogine indicator, the average minimum travel time, and the average minimum specific travel time.

As a result of testing of the subsystem for model calculations, data of the $n$ and Tm parameters were obtained, which characterize the quality of traffic management in this section.

Having obtained the results of calculating the parameters of the Herman-Prigogine model for specific streets of the city of Minsk, the points of the minimum average specific travel time $T_{m}$ and the indicator $\mathrm{n}$ of Herman-Prigogine for these streets were plotted in the graph (Figure 3 ). As seen here, the points are located irregularly, forming street clusters according to the quality of traffic management.

The FOREL algorithm was chosen for the clustering of this data set, since the number of clusters that should be obtained at the output was not known in advance. This algorithm requires specifying only the initial size of the expected clusters. By specifying the radius experimentally, the obtained output was a list of points with automatically generated cluster classes. The obtained clusters with a radius of 7 units are shown in Figure 4.

The illustration shows that the routes were divided into 6 clusters, each with a different number of streets. The parameters of each cluster are given in Table 1.

A brief description of road conditions is given for each cluster, which was determined on the basis of comparison with the indicators of other cities. Comparing the indicators of different cities, a conclusion can be made on the characteristics 
Table 2 Distribution of the streets of Minsk by clusters

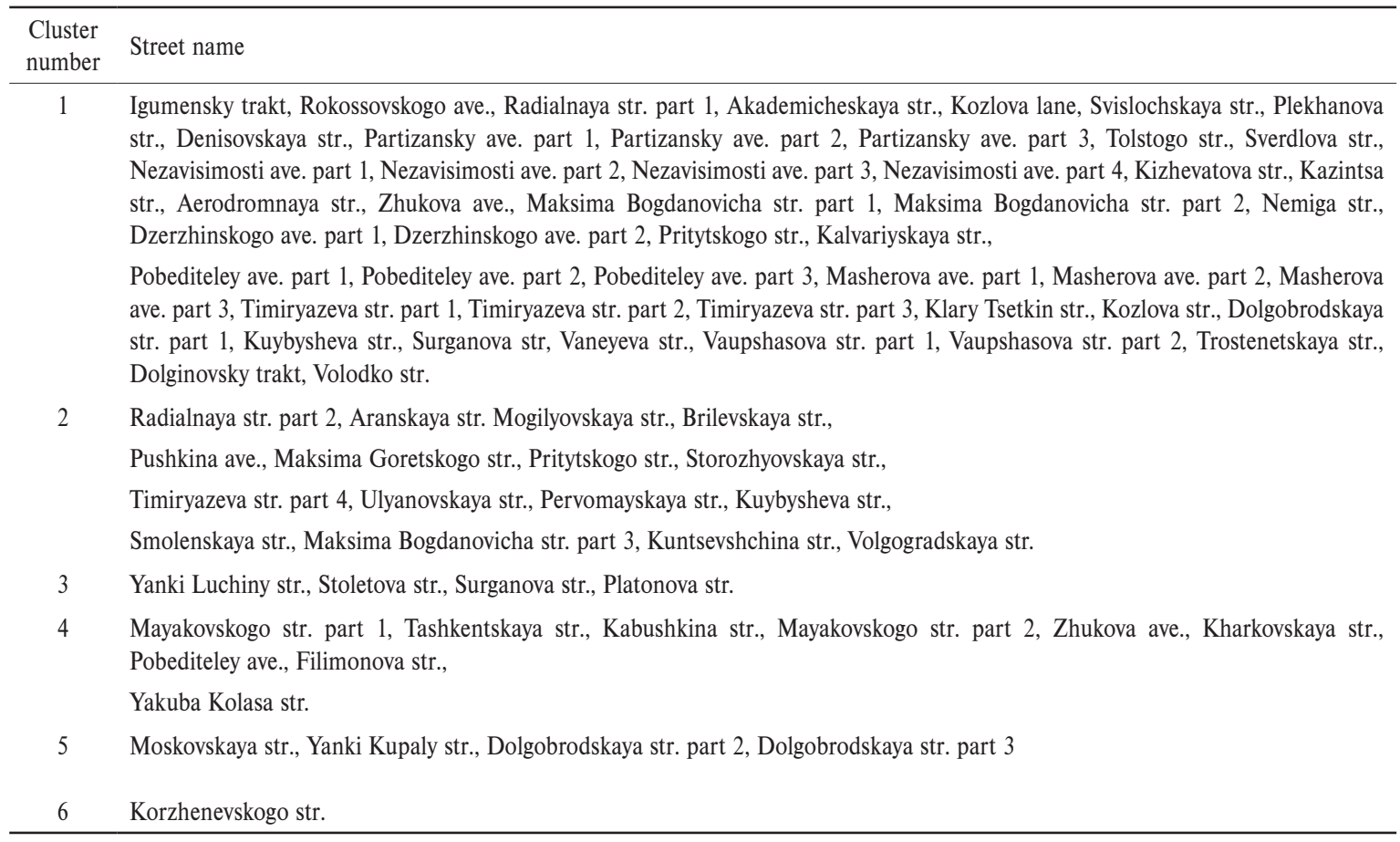

of traffic management in Minsk. Also, the degree of influence of the traffic flow load on the quality of service was determined in the "Brief description" column of Table 1.

The resulting route clustering was applied when plotting the routes on the map in different colors depending on the route class. This allows visually identifying road sections with different road conditions. The visualization results are shown in Figure 5.

The distribution of the streets of Minsk by clusters is given in Table 2.

Thus, the classification of the streets of Minsk according to the quality of traffic management was obtained, problematic streets were identified, and the results were visualized.

\section{Conclusions}

Mathematical modeling plays an increasingly importan role in methodological support of the development and implementation of any measures to manage the road network capacity. The application of mathematical models in the planning of management activities in the field of transport offers an opportunity:

- to assess the quality of traffic management;

- to predict the operational efficiency of management activities and their combinations based on the obtained estimates;

- to predict possible negative consequences of the implementation of management activities.

These predictions and estimates allow significantly improving the quality of management decisions in the transport sector.

The widespread introduction of navigation systems in transport opens up wide possibilities for collecting information on traffic flows. There are mathematical tools that continue to improve, which provide a solution for current problems of transport modeling and the assessment of traffic flow parameters.
Thus, for successful application of mathematical modeling methods for the transport system, recommendations for transport modeling, as well as programs for optimizing the road network capacity should be developed.

As part of this work, a software system has been developed that includes modules for collection of navigational data, storage and verification of track data, management of street and road data, calculation of the Herman-Prigogine model indicators, and classification of streets according to these parameters on the basis of passive processing of navigational data on vehicle traffic on transport highways and street and roads networks of cities.

The main advantages and novelty of the developed system are as follows:

- cloud computing (using to store large amounts of cloud storage data);

- availability of a mobile client application for the Android OS for collecting data in addition to the ability to work with already collected navigation data from the navigation and information center and other sources;

- support for formats of modern GPS trackers;

- use for calculating and processing the data of modern web technologies;

- scalability and customizability of the system.

As a result of testing of the developed software, results confirming the working efficiency of the proposed algorithms were obtained. Also, as a result of using the navigational data collected in the Republic of Belarus, in particular in the city of Minsk, the ability of the software system to distinguish street classes with different conditions of traffic management and the influence of the traffic flow load on the capacity was demonstrated.

The obtained results of the assessment of traffic flow parameters can be used to improve the efficiency and quality of the activities of state bodies, services, and companies in the transport sector in order to support the decision-making on accounting and 
redistribution of traffic flows within transport highways and street and road networks, on analyzing the transport load, on providing optimal traffic management, and when upgrading the existing road networks and designing new road networks. Moreover, this tool can be used to analyze transport corridors, main highways, and city streets in order to find sections that primarily require upgrading and improving.

\section{References}

[1] EBOLI, L., MAZZULlA, G., PUNGILlO, G. Measuring the driver's perception error in the traffic accident risk evaluation. IET Intelligent Transport Systems [online]. 2017, 11(10), 659-666. ISSN 1751-956X/eISSN 1751-9578. Available from: https://doi. org/10.1049/iet-its.2017.0084

[2] PERSIA, L. et al. Strategies and measures for sustainable urban transport systems. Transportation Research Procedia [online]. 2016, 14, p. 955-964. ISSN 2352-1465. Available from: https://doi.org/10.1016/j.trpro.2016.05.075

[3] GALKIN, A., et al. Perspective of decreasing of road traffic pollution in the cities. In: International Multidisciplinary Scientific GeoConference Surveying Geology and Mining Ecology Management SGEM 2018 : proceedings [online]. Vol. 18(4.2). Albena, Bulgaria, 2018. ISBN 978-619-7408-45-4, ISSN 1314-2704, p. 547-554. Available from: https://doi.org/10.5593/sgem2018/4.2/ S19.071

[4] Material design - Google design guidelines [online]. Avaliable from: https://www.google.com/design/spec/material-design/ introduction.html

[5] PONKRATOV, D., et al. Traffic streams and anti-congestions activities in city networks. International Journal of Automation, Control and Intelligent Systems [online]. 2015, 1(2), 21-26. ISSN 2381-7526/eISSN 2381-7534. Available from: http://files. aiscience.org/journal/article/html/70100016.html

[6] DREW, D. R. Traffic flow theory and control. New York: McGraw-Hill, 1968. ISBN 978-0070178311.

[7] VRUBEL, Y. A. Kapskiy, D. V., Kot, Y. N. The definition of losses in road traffic: a monograph (in Russian). Minsk: BNTU, 2006.

[8] EBOLI, L., et al. Experimental relationships between operating speeds of successive road design elements in two-lane rural highways. Transport [online]. 2015, 32(2), p. 138-145. ISSN 1648-4142/eISSN 1648-3480. Available from: https://doi.org/10.3846 / 16484142.2015 .1110831

[9] LIGHTHILL, M. J., WHITHAM, G. B. On kinematic waves II. A theory of traffic flow on long crowded roads. Proceedings of the Royal Society A: Mathematical, Physical and Engineering Sciences [online]. 1955, 229(1178), p. 281-345. ISSN 0080-4630/eISSN 2053-9169. Available from: https://doi.org/10.1098/rspa.1955.0089

[10] RICHARDS, P. I. Shock waves on the highway. Operations Research [online]. 1956, 4(1), p. 42-51. ISSN 0030-364X/eISSN 15265463. Available from: https://doi.org/10.1287/opre.4.1.42

[11] GARTNER, N. H. MESSER, C. J. RATHI, A. K., eds. Traffic flow theory: A state-of-the-art report. Washington DC: Transportation Research Board, 2001.

[12] PAYNE, H. J. Models of freeway traffic and control. In: Bekey, G. A., ed., Mathematical Models of Public Systems. La Jolla, CA: Simulation Council, Inc. 1971, 1, p. 51-61.

[13] DAGANZO, C. F. Fundamentals of transportation and traffic operations. 1. ed. New York: Elsevier Science inc., 1997. ISBN 9780080427850

[14] SHVETSOV, V. I. Mathematical modeling of traffic flows (in Russian). Avtomatika i telemekhanika. 2003, 11, p. 3-46. ISSN 00052310.

[15] HERMAN, R., PRIGOGINC, I. A two-fluid approach to town traffic. Science [online]. 1979, 204(4389), p. 148-151. ISSN 00368075/eISSN 1095-9203. Available from: https://doi.org/10.1126/science.204.4389.148

[16] NELSON, P., SOPASAKIS, A. The prigogine-herman kinetic model predicts widely scattered traffic flow data at high concentrations. Transportation Research Part B: Methodological [online]. 1998, 32(8), p. 589-604. ISSN 0191-2615. Available from: https://doi. org/10.1016/S0191-2615(98)00020-4

[17] GECCHELE, G., et al. Data mining methods for traffic monitoring data analysis: A case study. Procedia-Social and Behavioral Sciences [online]. 2011, 20, p. 455-464. ISSN 1877-0428. Available from: https://doi.org/10.1016/j.sbspro.2011.08.052

[18] DERRMANN, T., et al. Estimating urban road traffic states using mobile network signaling data. In: IEEE 20th International Conference on Intelligent Transportation Systems ITSC 2017 : proceedings. 2017. eISSN 2153-0017, p. 1-7. Available from: https:// doi.org/ 10.1109/ITSC.2017.8317718

[19] PRIGOGINE, I., HERMAN R. Kinetic theory of vehicular traffic. New York: American Elsevier Publishing Company, 1971.

[20] Blinkin, M. Ya., Tkachenko, B. A. Systemassessment of traffic conditions on the basis of the Herman-Prigogine [Sistemnaya otsenka uslovii dvizheniya na baze modeli Hermana-Prigozhina]. Sotsial'no-ekonomicheskie problem razvitiya transportnyh system gorodov i zon ih vliyaniya, 2009 [Electronic resource]: http://www.waksman.ru/Russian/Org\&B/2008/blinkin1.htm. Last accessed: 1.03.2019

[21] Google Maps Javascript API - Google Developers [online]. Avaliable from: https://developers.google.com/maps/documentation/ javascript/examples/event-simple 
[22] Algorithms family FOREL (in Russian) - Wikipedia [online]. Avaliable from: https://ru.wikipedia.org/wiki/Algoritmy_semeystva_ FOREL

[23] jQuery API Documentation - jQuery [online]. Avaliable from: http://api.jquery.com/

[24] Matplotlib 1.5.0 documentation [online]. Avaliable from: http://matplotlib.org/contents.html

[25] SQLite Documentation [online]. Avaliable from: https://www.sqlite.org/docs.html

[26] Flask (A Python Microframework) [online]. Avaliable from: http://flask.pocoo.org/

[27] Location and Sensors APIs - Android developers [online]. Avaliable from: http://developer.android.com/guide/topics/sensors/ index.html.

[28] SQLAlchemy - The Database Toolkit for Python [online]. Avaliable from: http://www.sqlalchemy.org/

[29] Web "Flask (A Python Microframework)" [online]. Avaliable from: http://flask.pocoo.org/

[30] LUTZ, M. Learning Python (in Russian). 4. ed. Sebastopol, CA: O’Reilly Media, Inc. 2010. ISBN 978-0-596-15806-4.

[31] Parse Android Developers Guide - Web "Parse" [online]. Avaliable from: https://parse.com/docs/android/guide 